Egyptian Journal of Aquatic Biology \& Fisheries

Zoology Department, Faculty of Science,

Ain Shams University, Cairo, Egypt.

ISSN $1110-6131$

Vol. 25(2): 457 - 464 (2021)

www.ejabf.journals.ekb.eg

\title{
Oil Spill Cleanup Using Chemically Modified Natural Fibers: Trial for Practical Application
}

Ola Abdelwahab ${ }^{1 *}$, Walaa M. Thabet ${ }^{1}$, Samir M. Nasr $^{2}$, Seifeldin Nafea $^{3}$

1. National Institute of Oceanography and Fisheries, NIOF, Egypt

2. Institute of Graduate Studies and Research, Egypt

3. Zewail City of Science and Technology, Egypt

*Corresponding author: olaabdelwahab53@ hotmail.com.

ARTICLE INFO

Article History:

Received: Nov. 26, 2020

Accepted: March 22, 2021

Online: April 15, 2021

Keywords:

Oil spill, sorption, field application, natural fibers.

\begin{abstract}
An oil spill was simulated in a basin connected to the sea, which presents inside the National Institute of Oceanography and Fisheries. Two available natural fibrous materials were used after modification as sorbent materials for the treatment of oil spills. Sorption capacities of LCLA and PFLA onto crude oil were preliminarily examined in the laboratory before applying in the field. An amount of LCLA and PFLA were wrapped in a polyester circular pad. Cylindrical-shaped acrylic vessels, with perforated sides, were fixed with cork sheets to keep them floating on the water surface. $1.1 \mathrm{~mm}$ thickness of light crude oil was spilled inside the acrylic vessels. LCLA and PFLA containing pads were then dipped into an oil layer floating on seawater. Pads were laid into the interface between oil and seawater for $30 \mathrm{~min}$ (one pad per each vessel). Sorption capacities were measured as 11.0 and 9.8 as gram oil per gram of sorbent for LCLA and PFLA, respectively. These results were close to the sorption capacities produced in the laboratory using the same oil and sorbent materials indicating that the tested sorbents have a cheerful application in the real field.
\end{abstract}

\section{INTRODUCTION}

Oil pollution represents a visible and most significant case of marine pollution. During the last decade, approximately thousand tons of oil are spilled by tankers annually worldwide (Karakasi and Moutsatsou, 2010; Chang et al., 2014; Michel and Fingas, 2016). Immediate action for preventing of oil pollution is required and to this direction several oil spill clean-up techniques. Various methods have been used for controlling oil spills, minimizing their impact on human health and the environment. (Delaune et al., 1999; Lessard and Demarco, 2000; Hussein et al., 2011; Elkady et al., 2015) 
The seriousness of oil spills through accidents depends on several factors, such as the amount of spilled oil, the final destination of the spilled oil, the weather and the season of the year (Toyoda and Inagaki, 2000; Zamparas et al., 2020).

In order to treat a massive oil spill, quick and large scale skimming is required; one uses sorbents with high sorption capacities and rates. In the case of oil spill in water, the sorbent materials used must be hydrophobic so they can adsorb oil only without water. In order to protect the aquatic environment from pollution, sorbents that have a large capacity and easy manipulation are needed (Abdelwahab et al., 2017).

Natural fibers are among the alternatives for oily wastewater treatment for their simplicity, excellent oil removal properties, environmentally friendly characteristics and easy availability and feasibility (Shi $\boldsymbol{e t}$ al., 2019; Do et al., 2020; El-Gheriany $\boldsymbol{e t}$ al., 2020). It was found that investments in natural sorbents as a sustainable oil spill cleanup technique will overcome the limitations of existing cleanup techniques (Wahi et al., 2013; Kyzas and Kostoglou, 2014). In this study, the potential of organically modified fibers of Lauffa Cylindrica and Palm Fibers to remove crude oil was investigated to provide an easily, eco-friendly and cost effective method of cleaning oil spills and oil recovery. Meanwhile, sorbent Pads were designed to control and clean up spills in small closed aquatic areas. They were made of fabric which can repel water and absorb only petroleum-based fluids to ensure efficiency in the cleanup operation. Oil Pads can be used to catch leaks, drips and spills or use them outdoors to soak up oil sheen off the surface of water.

\section{MATERIALS AND METHODS}

\section{Preparation of sorbent}

Two available natural fibrous materials were used after modification as sorbent materials for treatment of oil spill. The fibers of luffa cylindrical and palm fibers were collected from the local market in Egypt. Fibers were treated after cleaning and classifying in a standard set of sieves to uniform particle size. Lauric acid solution of $1 \mathrm{M}$ concentration was used for sorbents modification. Fibers were suspended in the acid solution while the stirred for $6 \mathrm{~h}$ and then it was filtered. The resultant adsorbent sample was washed with n-hexane several times before being oven-dried overnight. The resultant treated fibrous sorbents were labeled as LCLA and PFLA for treated luffa cylinderica and treated palm fibers, respectively.

\section{Sorption laboratory experiments}

To study the capacity of sorbents. A calculated volume of crude oil was poured into a $250-\mathrm{ml}$ beaker containing $100 \mathrm{ml}$ of sea water at different oil film thicknesses $(1.1,2.3$ and $3.4 \mathrm{~mm}$ ). $0.2 \mathrm{~g}$ of adsorbent with grain size $>0.2 \mathrm{~mm}$, was added to the oil-water mixture as the method of Abdelwahab (Abdelwahab et al., 2014; Abdelwahab et al., 2017). Shaking was persisting for an hour at $140 \mathrm{rpm}$, while samples withdrawn 
according scheduled time interval of $(1,5,10,15,30,60 \mathrm{~min})$. The sample weight was determined and capacity of oil was calculated at time intervals using the following relation

Sorption capacity $(\mathrm{g} / \mathrm{L})=\frac{\text { mass of oil removed }}{\text { mass of sorbent }}$

\section{Field application experiment}

To simulate a real oil spill in the field, the study was crafted in a basin connected to the sea present in the National Institute of Oceanography and Fisheries, NIOF, Alexandria. Crude oil was spilled inside cylindrical-shaped acrylic vessels floating on the surface of sea water. The vessels had a diameter of $26 \mathrm{~cm}$ and were fixed with cork sheets to make sure of their buoyancy. The cork sheets keep them floating on water surface. All cylinders were opened from both sides, which facilitated seawater exchanges between interior and exterior sides of the vessels. A Pre-calculated volume of oil was poured inside each vessel to attain $1.1 \mathrm{~mm}$ thickness of the oil film. Stoichiometric amounts of LCLA and PFLA were wrapped in a polyester circular pad $(25.5 \mathrm{~cm}$ diameter) where it was subjected to shaking by the natural waves of water basin. Each pad was introduced to the interface between oil and sea water for $15 \mathrm{~min}$ (one pad/ vessel). After that, the pads were detached and the amount of adsorbed oil was determined. After which, sorption capacity of LCLA and PFLA was calculated and compared with the one obtained in the laboratory.

\section{RESULTS AND DISCUSSION}

\section{Effect of Thickness and Time on Sorption Capacity}

Figs. 1 and 2 represent sorption capacities of LCLA and PFLA as a function of the time using different thicknesses of crude oil, respectively. The used oil viscosity was $62 \mathrm{cp}$ and its density $85 \mathrm{~g} / \mathrm{cm}^{3}$. It is obvious that the sorption capacity increases with the increase in the sorption time until it reaches a maximum capacity of $18.0 \mathrm{~g}$ oil $/ \mathrm{g}$ of sorbent using LCLA after 60 min shaking time, while it reaches $17 \mathrm{~g}$ oil/g of sorbent by using PFLA.

Meanwhile, it was found that the sorption capacity increases with the increase in oil film thickness. These results are in agreement with the previous studies, as they discussed effect of contact time on oil adsorption capacity using activated recycled rubber, date palm powder, low grade raw cotton fibers, coconut, raw bagasse and barely straw, respectively (Said et al., 2009; Hussein et al., 2009; Aisien and Aisien, 2012; AlZubaidy, 2012; Okafor et al., 2012). 


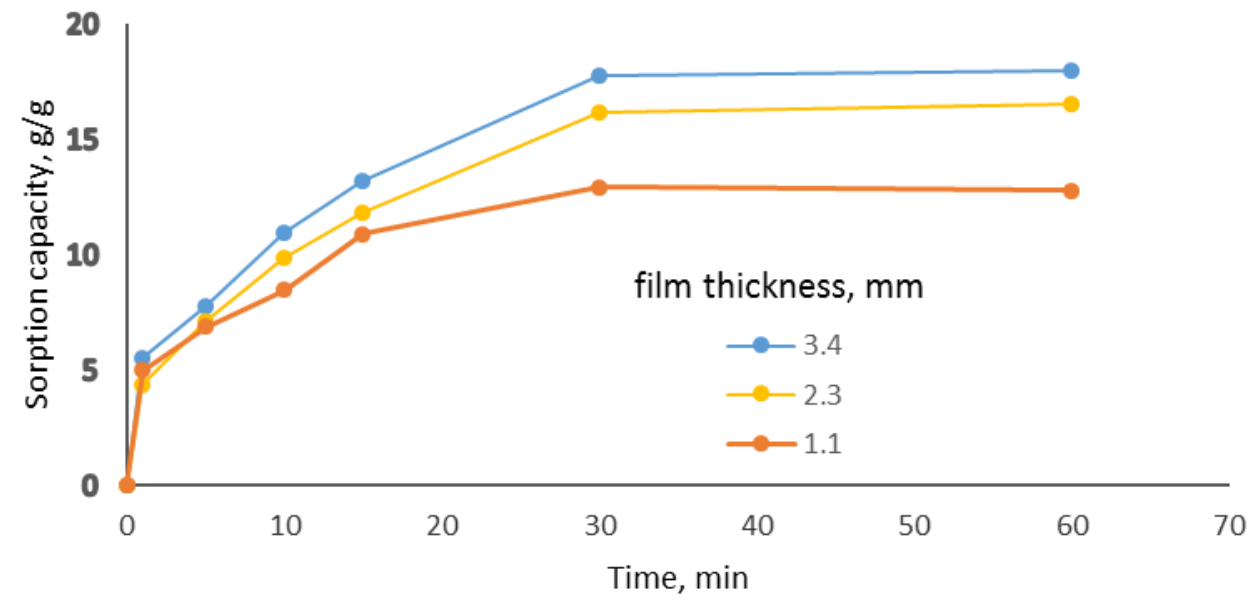

Fig. 1. Sorption capacities of LCLA as a function of time exposed to different crude oil film thicknesses

Due to the addition of LCLA and PFLA onto the crude oil floating on the water, it was noticed that after 60 min shaking the layer of oil disappeared in case of using LCLA due to its sorption by the adsorbent while, the oil layer became less dense after the use of PFLA. Typical photographs of the appearance of the crude oil floating layer before and after the addition of LCLA and PFLA onto the oil surface are shown in Fig. 3. The complete disappearance of the oil layer after using LCLA refers to its higher sorption efficiency than PFLA

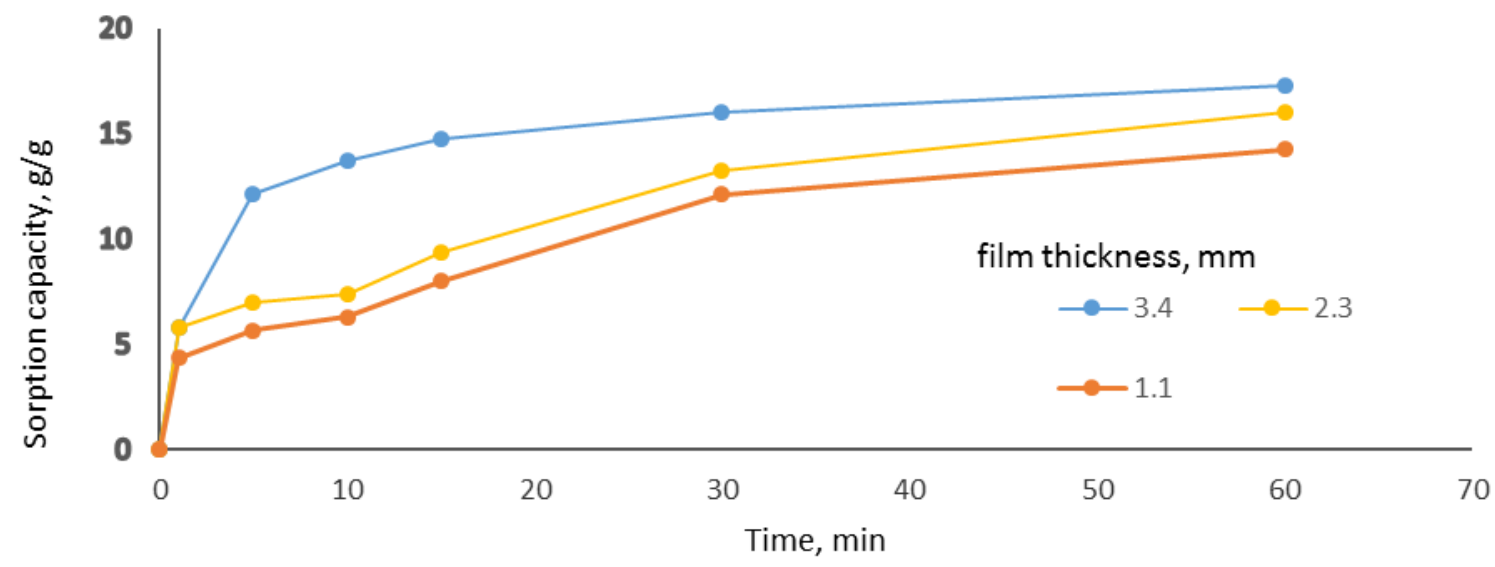

Fig. 2. Sorption capacities of PFLA as a function of the time exposed to different crude oil film thicknesses 


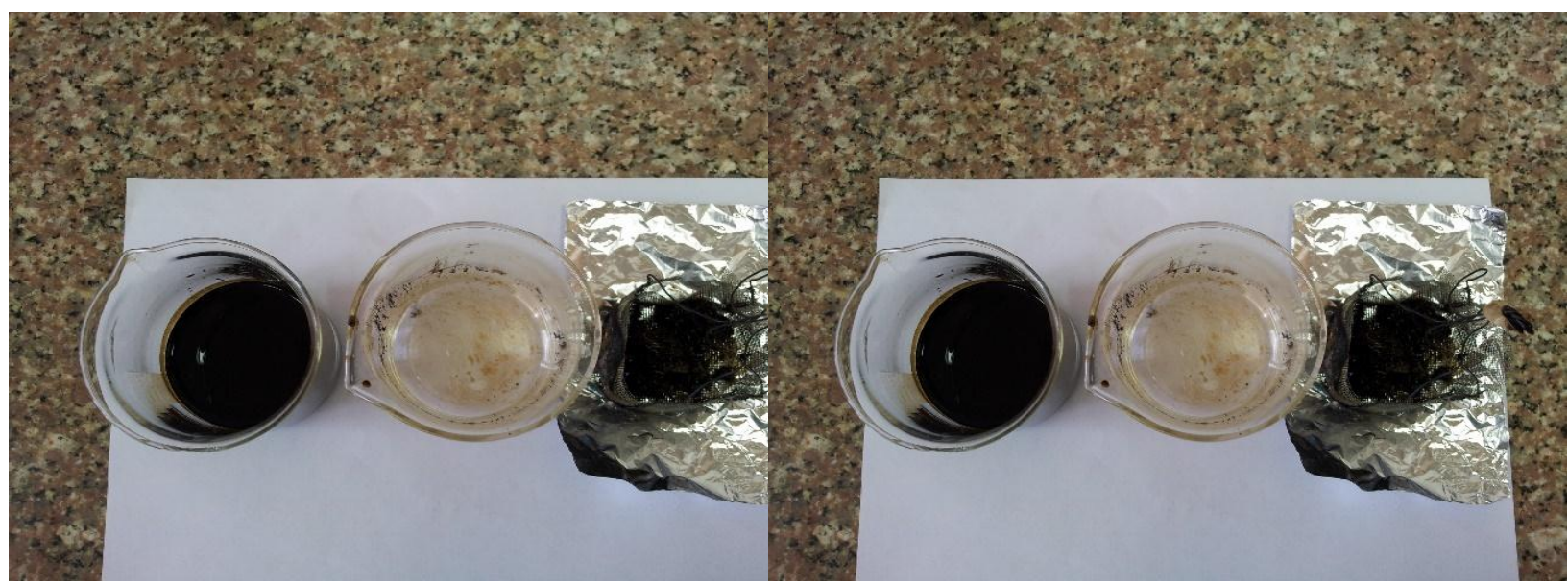

(a)

(b)

Fig.3. Typical photographs of the crude oil floating layer before and after the addition of (a) LCLA and (b) PFLA

\section{Sorption of Oil Spill- Simulated Experiment}

Fig. 4 represents a test simulates the actual spill in the environment. The experiment was built in a basin connected to the sea. In the beginning, the oil was spread on the water surface inside the cylindrical-shaped acrylic vessels to attain $1.1 \mathrm{~mm}$ thickness of the oil film, as shown in Fig 4a. Then, two polyester circular pads containing LCLA and PFLA, individually, were immersed in the oil layer at the interface between oil and sea water to attain good contact between the pad and oil, as shown in Fig 4b. The pads were left for 30 min floating on the oil surface layer, they were then removed for analysis. Fig $4 \mathrm{c}$ and Fig. $4 \mathrm{~d}$ indicate the surface and internal appearance of the LCLA and PFLA pads after sorption, respectively. The pads look saturated with crude oil.
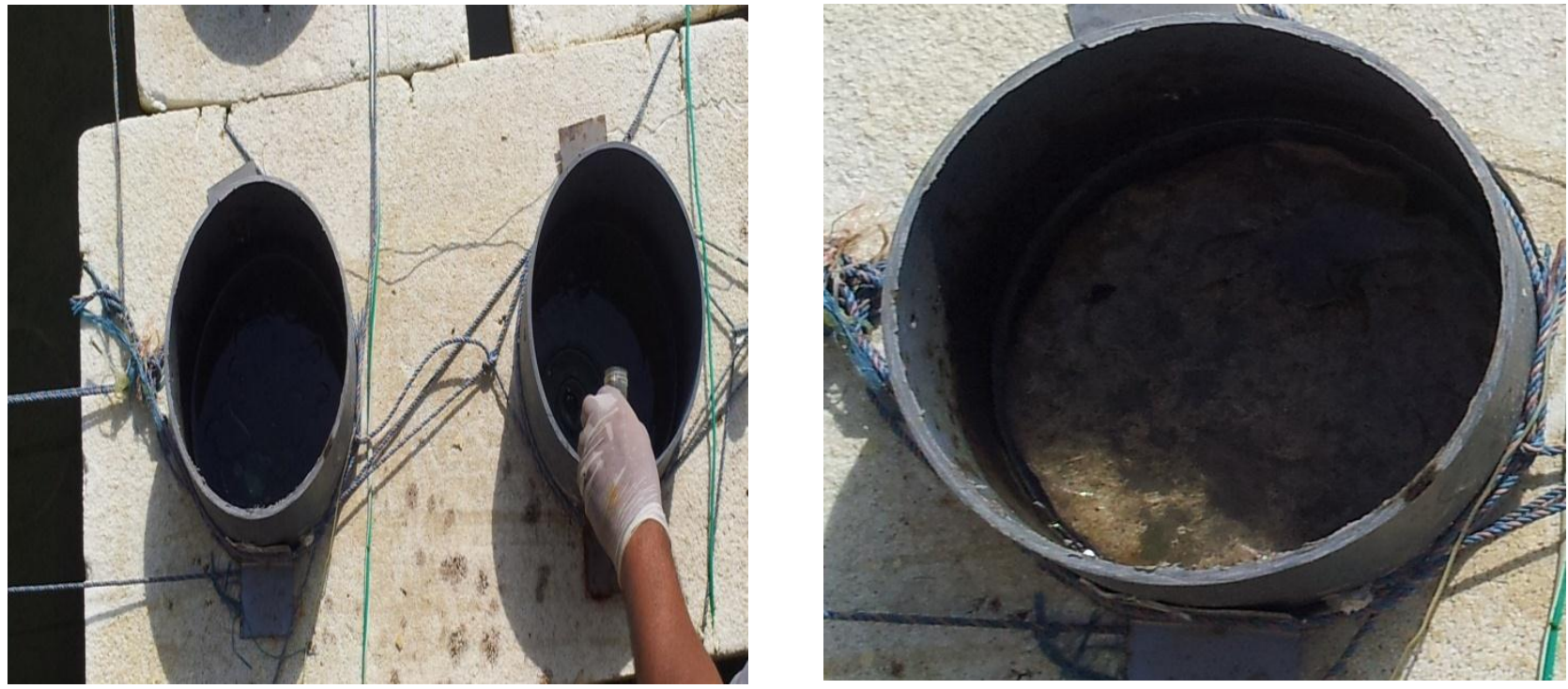
a.

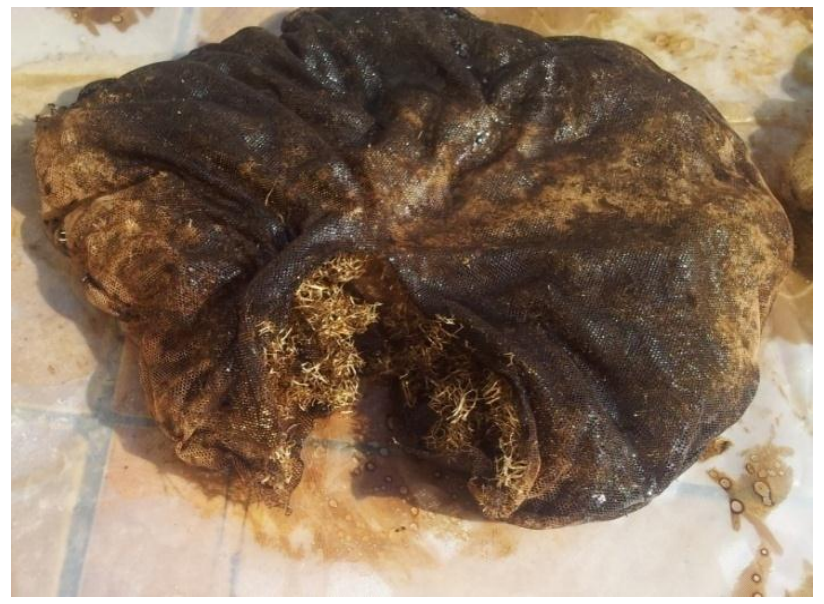

c. b.

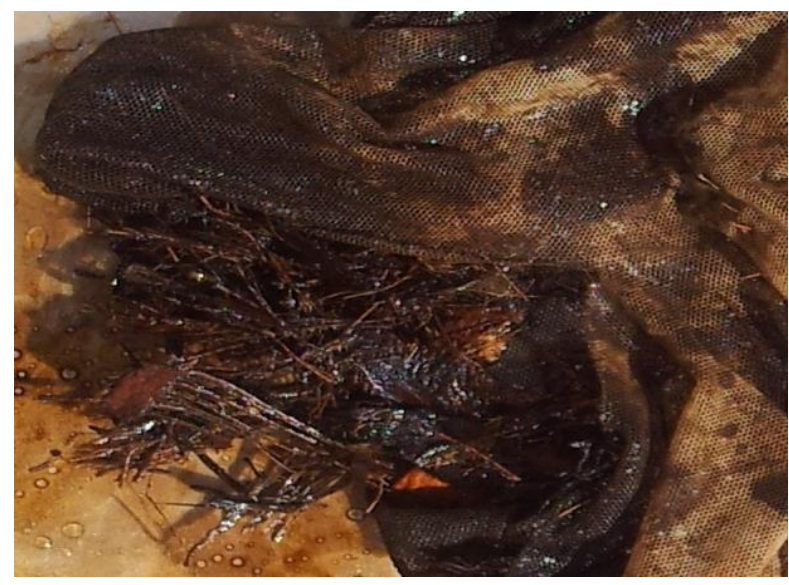

d.

Fig. 4 (a) calculated amount of oil is powered inside each tube. (b) pad immediately after added to the oil/water interface. (c) and (d) surface and internal appearance of the LCLA and PFLA pads after sorption

Pads acted as a large sponge that adsorbed the oil from the water. Capacities were measured as 11.0 and 9.8 gram oil per gram of sorbent for LCLA and PFLA, respectively. These results were close to the capacities produced in the lab after $30 \mathrm{~min}$ shaking, indicating that the tested sorbents have promising application in the field.

\section{CONCLUSION}

Two available natural fibrous materials were used after modification as sorbent materials for treatment of oil spill. The sorption capacity of LCLA and PFLA was studied first in the laboratory. Sorption capacity of the fibers was increased with time and thickness of oil film. LCLA and PFLA were wrapped in a polyester circular pad. The pads exhibited excellent sorption capacity for oils, under simulated field conditions (sea water in NIOF basin with the oil layer). Pads adsorbed the oil spill from the water. Pads sorption capacities were measured as 11.0 and 9.8 gram oil per gram of sorbent for LCLA and PFLA, respectively. These results were nearly close to the capacities produced in the lab after 30 min shaking. Based on the results obtained, the pads containing LCLA and PFLA have excellent commercial potential as a sorbent for oil.

There is as yet little information containing a field study of comparison between different sorbents. Although much has been accomplished in the area of low-cost sorbents, much work is necessary to (i) predict the performance of the adsorption processes for oil spill removal from simulated spills under a range of operating conditions, (ii) better understand adsorption mechanisms, and (iii) demonstrate the use of inexpensive bio-sorbents at real field applications. 


\section{REFERENCES}

Abdelwahab, O. (2014). Assessment of raw luffa as a natural hollow oleophilic fibrous sorbent for oil spill cleanup. Alex. Eng. J., 53: 213-218.

Abdelwahab, O.; Nasr, S. M. and Thabet, W. M. (2017) Palm fibers and modified palm fibers adsorbents for different oils. Alex. Eng. J, 56(4): 749-755.

Aisien, F.A. and Aisien, E.T. (2012) Application of activated recycled rubber from used tyres in oil spill cleanup, Turkish J. Eng. Environ. Sci., 36: 171-177.

Al-Zubaidy, E. (2012) Effect of activation of date palm kernel powder on the remediation process of oil polluted water, Int. J. Environ. Pollut. Remediation. 1: 38-42.

Chang, S.E.; Stone, J.; Demes, K. and Piscitelli, M. (2014) Consequences of oil spills: A review and framework for informing planning. Ecol. Soc., 19(2): 26. http://dx.doi.org/10.5751/ES-06406-190226

Delaune, R. D.; Lindau, C. W. and Jugsujinda A. (1999). "Effectiveness of "Nochar" solidifier polymer in removing oil from open water in coastal wetlands" Spill Sci Technol Bull., 5(5/6): 357-9.

Do, N.H.N.; Luu, T.P.; Thai, Q.B.; Le, D.K.; Chau, N.D.Q.; Nguyen, S.T.; Le, P.K.; Phan-Thien, N. and Duong, H.M. (2020) Advanced fabrication and application of pineapple aerogels from agricultural waste. Mater. Technol., 35: 807-814.

El-Gheriany, I.A.; Ahmad El-Saqa, F.; Abd El Razek Amer, A. and Hussein, M. (2020). Oil spill sorption capacity of raw and thermally modified orange peel waste. Alex. Eng. J., 59: 925-932.

Elkady, M. F.; M. Hussien M. and Abou-rady R. (2015) "Equilibrium and kinetics behavior of oil spill process onto synthesized nano-activated carbon". Am. J. Appl. Chem., 3: 22-30.

Hussein, M.; Amer, A. A. and Sawsan, I. L. (2011). Heavy oil spill cleanup using law grade raw cotton fibers: Trial for practical application. J. Pet. Technol. Altern. Fuels, 2(8): 132-140.

Hussein, M.; Amer, A.A.; El-Maghraby A., and Taha A., (2009) Availability of barley straw application on oil spill cleanup. Int. J. Environ. Sci. Technol., 6: 123-130.

Karakasi, O. K. and Moutsatsou A. (2010). "Surface modification of high calcium fly ash for its application in oil spill cleanup,"Fuel., 89: 3966-3970. 
Kyzas, G. and Kostoglou, M. (2014). Green Adsorbents for Wastewaters: A Critical Review. Materials, 7: 333-364.

Lessard, R. R. and Demarco, G. (2000). "The significance of oil spill dispersants. Spill SciTechnol Bull. Vol. 6(1): 59-68.

Michel, J. and Fingas, M. (2016). Oil Spills: Causes, Consequences, Prevention, and Countermeasures. In Fossil Fuels: Current Status and Future Directions; WSPC: Singapore, 2016; 159-201. ISBN 9789814699983.

Okafor P.C.; Okon, P.U.; Daniel, E.F. and Ebenso, E.E. (2012) Adsorption capacity of coconut (Cocosnucifera L.) shell for lead, copper, cadmium and arsenic from aqueous solutions, Int. J. Electrochem. Sci., 7: 12354-12369.

Said, A.A.; Ludwick, A.G. and Aglan, H.A. (2009) Usefulness of raw bagasse for oil absorption: a comparison of raw and acylated bagasse and their component. Bioresour. Technol., 100: 2219-2222.

Shi, G.; Qian, Y.; Tan, F.; Cai,W.; Li, Y. and Cao, Y. (2019) Controllable synthesis of pomelo peel-based aerogel and its application in adsorption of oil/organic pollutants. R. Soc. Open Sci., 6; 181823. doi.org/10.1098/rsos.181823

Toyoda, M. and Inagaki, M. (2000). Heavy oil sorption using exfoliated graphite New application of exfoliated graphite to protect heavy oil pollution. Carbon. 38: $199-210$.

Wahi, R.; Chuah, L. A.; Choong, T. S. Y.; Ngaini, Z. and Nourouzi, M. M. (2013). Oil removal from aqueous state by natural fibrous sorbent: An overview. Sep. Purif. Technol., 113: 51-63.

Zamparas, M.; Tzivras, D.; Dracopoulos V. and T. Ioannides, (2020). Application of Sorbents for Oil Spill Cleanup Focusing on Natural-Based Modified Materials: A Review. Moleculoles., 25: 4522; doi:10.3390/molecules25194522. 